\title{
On the postcritical set of a rational map
}

\author{
Laura G. DeMarco, Sarah C. Koch and Curtis T. McMullen
}

20 September 2017

\begin{abstract}
The postcritical set $P(f)$ of a rational map $f: \mathbb{P}^{1} \rightarrow \mathbb{P}^{1}$ is the smallest forward invariant subset of $\mathbb{P}^{1}$ that contains the critical values of $f$. In this paper we show that every finite set $X \subset \mathbb{P}^{1}(\overline{\mathbb{Q}})$ can be realized as the postcritical set of a rational map. We also show that every map $F: X \rightarrow X$ defined on a finite set $X \subset \mathbb{P}^{1}(\mathbb{C})$ can be realized by a rational map $f: P(f) \rightarrow P(f)$, provided we allow small perturbations of the set $X$. The proofs involve Belyi's theorem and iteration on Teichmüller space.
\end{abstract}

\section{Contents}

1 Introduction . . . . . . . . . . . . . . 1

2 Every finite set of algebraic numbers is a postcritical set . . . 2

3 Contraction on Teichmüller space . . . . . . . . . . . . . 4

$4 \quad$ Prescribed dynamics on $P(f) \ldots \ldots \ldots$. . . . . . 7

5 Solution to a Hurwitz problem . . . . . . . . . . . 9

6 Prescribed critical points in $P(f) \ldots \ldots$. . . . . . . . . . . . . .

$7 \quad$ Alternative constructions . . . . . . . . . . 17

Research supported in part by the NSF. 


\section{Introduction}

Let $f: \mathbb{P}^{1} \rightarrow \mathbb{P}^{1}$ be a rational map on the Riemann sphere $\mathbb{P}^{1}=\mathbb{P}^{1}(\mathbb{C})$ of degree $d \geq 2$. Let $C(f) \subset \mathbb{P}^{1}$ denote the set of critical points of $f$, and let $V(f)$ denote the set of critical values. The postcritical set of $f$ is defined by

$$
P(f)=\bigcup_{n \geq 0} f^{n}(V(f)) .
$$

A rational map is postcritically finite if $|P(f)|<\infty$. Most postcritically finite rational maps are rigid, in the sense defined in $\$ 2$ f for example, $f$ is rigid if $|P(f)|>4$. When $f$ is rigid, it is conformally conjugate to a rational map $g$ defined over the algebraic closure of $\mathbb{Q}$, in which case we have $P(g) \subset \mathbb{P}^{1}(\overline{\mathbb{Q}})$. In this paper we prove the converse:

Theorem 1.1 For any finite set $X \subset \mathbb{P}^{1}(\overline{\mathbb{Q}})$ with $|X| \geq 2$, there exists a rigid, postcritically finite rational map such that $P(f)=X$.

We can also arrange that $P(f) \subset C(f)$, which implies that $f$ is hyperbolic. The proof ( 82 uses Belyi's theorem; consequently, the degree of the map $f$ we construct may be enormous, even when the set $X$ is rather simple.

In light of Theorem 1.1, we formulate the following more precise question about dynamics on the postcritical set.

Question 1.2 Let $X \subset \mathbb{P}^{1}(\overline{\mathbb{Q}})$ be a finite set. Is every map $F: X \rightarrow X$ realized by a rigid rational map $f: P(f) \rightarrow P(f)$ with $P(f)=X$ ?

It is easy to see the answer can be no when $|X|=2$, and it is yes when $|X|=3$ (for a proof, see $\$ 7$ ). We do not know of any negative answer when $|X|=4$, but most cases are simply open. For example, we do not know the answer for $X=\{0,1,4, \infty\}$ and $F(x)=x$.

We can, however, show that Question 1.2 has a positive answer if we allow perturbations of $X$ as a subset of $\mathbb{P}^{1}(\mathbb{C})$.

Theorem 1.3 Let $F: X \rightarrow X$ be an arbitrary map defined on a finite set $X \subset \mathbb{P}^{1}(\mathbb{C})$ with $|X| \geq 3$. Then there exists a sequence of rigid postcritically finite rational maps $f_{n}$ such that $\left|P\left(f_{n}\right)\right|=|X|$,

$$
P\left(f_{n}\right) \rightarrow X \quad \text { and } \quad f_{n}\left|P\left(f_{n}\right) \rightarrow F\right| X
$$

as $n \rightarrow \infty$. 
The proof (\$4) uses iteration on Teichmüller space, as in the proof of Thurston's topological characterization of postcritically finite rational maps [DH].

In $\$ 5$, we establish the following new Hurwitz-type result, which may be of interest in its own right:

Any collection of partitions $\mathcal{P}$ with $|\mathcal{P}| \geq 3$ can be extended to the passport $\mathcal{Q}$ of a rational map, with $|\mathcal{Q}|=|\mathcal{P}|$.

See Theorem 5.1. (Here the passport of a rational map $f$ is the collection of partitions of $\operatorname{deg}(f)$ arising from the fibers over its critical values.) We use this result to strengthen Theorem 1.3 in $\$ 6$ by showing that we can also specify the multiplicity of $F$ at each point of $X$.

Other constructions of rational maps with specified postcritical sets are presented in $\$ 7$.

\section{Every finite set of algebraic numbers is a post- critical set}

In this section we prove that any finite set $X \subset \mathbb{P}^{1}(\overline{\mathbb{Q}})$ with $2 \leq|X|<\infty$ arises as the postcritical set of a rigid rational map (Theorem 1.1). The result for rational maps follows easily from the following variant for polynomials.

Theorem 2.1 For any finite set of algebraic numbers $X \subset \mathbb{C}$ with $|X| \geq 1$, there exists a polynomial $f$ such that $P(f) \cap \mathbb{C}=X$.

Polynomials. To begin the proof, we remark that it is easy to construct polynomials with prescribed critical values. It is convenient, when discussing a polynomial $f$, to omit the point and infinity and let $C_{0}(f)=C(f) \cap \mathbb{C}$, and similarly for $V_{0}(f)$ and $P_{0}(f)$.

Lemma 2.2 For any finite set $X \subset \mathbb{C}$, there exists a polynomial $g$ with $V_{0}(g)=X$.

Proof. There are many ways to prove this result; for example, by induction on $|X|$, using the fact that $V_{0}(f \circ g)=V_{0}(f) \cup f\left(V_{0}(g)\right)$ and $V_{0}\left(z^{2}+a\right)=\{a\}$. 
(For a more precise result, see Corollary 5.4.)

Let us say $\beta$ is a Belyi polynomial if $V_{0}(\beta) \subset\{0,1\}$. We will also use following result from $[\mathrm{Bel}]$ :

Theorem 2.3 For any finite set $X \subset \overline{\mathbb{Q}}$, there exists a Belyi polynomial such that $\beta(X) \subset\{0,1\}$.

Proof of Theorem 2.1. Our aim is to construct a polynomial $f$ such that $P_{0}(f)=X$. This is easy if $|X| \leq 1$, so assume $|X| \geq 2$. Using Lemma 2.2, choose a polynomial $g$ such that $V_{0}(g)=X$, and precompose with an affine transformation so that $\{0,1\} \subset C_{0}(g)$. Let $\beta$ be a Belyi polynomial such that $\beta(X) \subset\{0,1\}$. Finally, let $f=g \circ \beta$.

We claim that $V_{0}(f)=X$. Indeed, we have

$$
V_{0}(f)=V_{0}(g \circ \beta)=V_{0}(g) \cup g\left(V_{0}(\beta)\right)=X \cup g\left(V_{0}(\beta)\right) ;
$$

but $V_{0}(\beta) \subset\{0,1\} \subset C_{0}(g)$, and therefore $g\left(V_{0}(\beta)\right) \subset X$, so $V_{0}(f)=X$. In particular $X \subset P_{0}(f)$. But in fact $P_{0}(f)=X$, since

$$
f(X)=g \circ \beta(X) \subset g(\{0,1\}) \subset X .
$$

Hyperbolicity. Note that

$$
P_{0}(f)=X \subset \beta^{-1}\left(C_{0}(g)\right) \subset C_{0}(g \circ \beta)=C_{0}(f)
$$

in the construction above. Thus every periodic point in $P(f)$ is superattracting, and hence $f$ is hyperbolic.

Rigidity. To deduce Theorem 1.1, we must first briefly discuss rigidity. A postcritically finite map $f: \mathbb{P}^{1} \rightarrow \mathbb{P}^{1}$ is rigid if any postcritically finite rational map $g$ uniformly close enough to $f$, and with $|P(g)|=|P(f)|$, is in fact conformally conjugate to $f$. For any fixed $d$ and $n$, the rigid maps with $\operatorname{deg}(f)=d$ and $|P(f)|=n$ fall into finitely many conjugacy classes.

By a theorem of Thurston $[\mathrm{DH}]$, the only postcritically finite rational maps $f$ that are not rigid are the flexible Lattès examples, which arise from the addition law on an elliptic curve [Mil2]. These flexible maps have $|P(f)|=4$ and Julia set $J(f)=\mathbb{P}^{1}$. Consequently, any postcritically finite rational map with a periodic critical point (such as a polynomial) is rigid.

Proof of Theorem 1.1. Let $X \subset \mathbb{P}^{1}(\overline{\mathbb{Q}})$ be a finite set with $|X| \geq 2$. After a change of coordinates defined over $\overline{\mathbb{Q}}$, we can assume that $\infty \in X$. Then by Theorem 2.1, there exists a polynomial $f$ with $P(f)=X$; and as we have just observed, any postcritically finite polynomial is rigid. 
Belyi degree and postcritical degree. Given a finite set $X \subset \overline{\mathbb{Q}}$, let $B(X)$ denote the minimum of $\operatorname{deg}(\beta)$ over all Belyi polynomials with $\beta(X) \subset$ $\{0,1\}$; and let $D(X)$ denote the minimum of $\operatorname{deg}(f)$ over all polynomials with $P_{0}(f)=X$.

Little is known about the general behavior of these degree functions (in particular, lower bounds seem hard to come by); however, the proof of Theorem 2.1 in concert with Corollary 5.4 gives the relation:

$$
D(X) \leq B(X)+|X|+1
$$

Both degree functions seem to merit further study.

\section{Contraction on Teichmüller space}

The rational maps $f$ constructed in the proof of Theorem 1.1 all satisfy $|f(P(f))| \leq 3$. We next address the problem of realizing more general dynamics on $P(f)$. Our construction will use iteration on Teichmüller space as in $[\mathrm{DH}]$. This section gives the needed background; for more details see [BCT, Hub].

Teichmüller spaces. Given a finite set $A \subset \mathbb{P}^{1}$ with $|A|=n$, we let $\mathcal{T}_{A} \cong \mathcal{T}_{0, n}$ denote the Teichmüller space of genus zero Riemann surfaces marked by $\left(\mathbb{P}^{1}, A\right)$.

A point in $\mathcal{T}_{A}$ is specified by another pair $\left(\mathbb{P}^{1}, A^{\prime}\right)$ together with an orientation-preserving marking homeomorphism:

$$
\phi:\left(\mathbb{P}^{1}, A\right) \rightarrow\left(\mathbb{P}^{1}, A^{\prime}\right) .
$$

Since $A^{\prime}=\phi(A)$, the marking $\phi$ alone determines a point $[\phi] \in \mathcal{T}_{A}$. Two markings $\phi_{1}, \phi_{2}$ determine the same point iff we can write $\phi_{2}=\alpha \circ \phi_{1} \circ \psi$, where $\alpha \in \operatorname{Aut}\left(\mathbb{P}^{1}\right)$ and $\psi$ is isotopic to the identity rel $A$.

The cotangent space to $\mathcal{T}_{A}$ at $\left(\mathbb{P}^{1}, A^{\prime}\right)$ is naturally identified with the vector space $Q\left(\mathbb{P}^{1}-A^{\prime}\right)$ consisting of meromorphic differentials $q=q(z) d z^{2}$ on $\mathbb{P}^{1}$ with at worst simple poles on $A^{\prime}$ and elsewhere holomorphic. The Teichmüller metric corresponds to the norm

$$
\|q\|=\int_{\mathbb{P}^{1}}|q|
$$

on the cotangent space.

Pullback. Now let $F: \mathbb{P}^{1} \rightarrow \mathbb{P}^{1}$ be a smooth branched covering map with $\operatorname{deg}(F) \geq 2$. The sets $C(F), V(F)$ and $P(F)$ are defined just as for a rational map. 
Consider a pair finite sets $A$ and $B$ in $\mathbb{P}^{1}$ such that

$$
F(A) \cup V(F) \subset B
$$

We then have a map of pairs

$$
F:\left(\mathbb{P}^{1}, A\right) \rightarrow\left(\mathbb{P}^{1}, B\right)
$$

that is branched only over $B$. By pullback of complex structures, we then obtain a holomorphic map

$$
\sigma_{F}: \mathcal{T}_{B} \rightarrow \mathcal{T}_{A}
$$

Contraction. Let $\sigma: \mathcal{T}_{B} \rightarrow \mathcal{T}_{A}$ be a holomorphic map between Teichmüller spaces. By the Schwarz lemma, $\|D \sigma\| \leq 1$ in the Teichmüller metric. We say $\sigma$ is contractive if $\|D \sigma\|<1$ at every point of $\mathcal{T}_{B}$. (We also say $\sigma$ is contractive if $|B|=3$, since then its image is a single point.) If $A=B$ and $\sigma$ is contractive, then $\sigma$ has at most one fixed point.

A branched covering $F$ is contractive if $\sigma_{F}$ is contractive.

The following result is well known and was a key step in the proof of Thurston's rigidity theorem for postcritically finite rational maps; see [DH, Prop. 3.3].

Proposition 3.1 The map $F$ is contractive if and only if there is no 4-tuple $B_{0} \subset B$ such that

$$
F^{-1}\left(B_{0}\right) \subset A \cup C(F) .
$$

Proof. We may assume $|B| \geq 4$. Suppose $\left\|D \sigma_{F}\right\|=1$ at some point in $\mathcal{T}_{B}$. The coderivative of $\sigma_{F}$ at this point is given explicitly by a pushforward map of the form

$$
f_{*}: Q\left(\mathbb{P}^{1}-A^{\prime}\right) \rightarrow Q\left(\mathbb{P}^{1}-B^{\prime}\right),
$$

where $f$ is a rational map of the same topological type as $F$. Since the domain of $f_{*}$ is finite dimensional, there exists a nonzero $q \in Q\left(\mathbb{P}^{1}-A^{\prime}\right)$ such that $\left\|f_{*} q\right\|=\|q\|$. The fact that there is no cancellation under pushforward implies that $q$ is a locally a positive real multiple of the pullback of $f_{*} q$. In fact, since $\left\|f^{*} f_{*} q\right\|=\operatorname{deg}(f)\left\|f_{*} q\right\|=\|q\|$, we must have

$$
f^{*} f_{*} q=\operatorname{deg}(f) q .
$$

Now recall that any meromorphic quadratic differential on $\mathbb{P}^{1}$ has at least 4 poles. Choose 4 points $B_{0} \subset B$ such that $\phi\left(B_{0}\right) \subset B^{\prime}$ is contained in the 
poles of $f_{*} q$. Then the equation above implies that the poles of $f^{*} f_{*} q$ lie in $A^{\prime}$. Thus any point in $F^{-1}\left(B_{0}\right)$ that does not lie in $A$ must be a critical point of $F$, giving condition (3.1) above.

For the converse, suppose we have 4 points $B_{0} \subset B$ satisfying (3.1). Consider, at any point in $\mathcal{T}_{B}$, a quadratic differential $q$ with poles only at the 4 points marked by $B_{0}$. Then $f^{*} q$ has poles only at points marked by $A$, and hence it represents a cotangent vector to $\mathcal{T}_{A}$. Since $\left\|f_{*}\left(f^{*} q\right)\right\|=$ $\operatorname{deg}(f)\|q\|=\left\|f^{*} q\right\|$, we have $\left\|D \sigma_{F}\right\|=1$ at every point in $\mathcal{T}_{B}$.

Example: dynamics. Let $f$ be a rational map with $|P(f)|=3$. Note that a rational map is a special case of a smooth branched covering. Consider a map of pairs

$$
f^{k}:\left(\mathbb{P}^{1}, A\right) \rightarrow\left(\mathbb{P}^{1}, B\right),
$$

such that $f^{k}(A) \cup P(f) \subset B$.

Proposition 3.2 The pullback map

$$
\sigma_{f^{k}}: \mathcal{T}_{B} \rightarrow \mathcal{T}_{A}
$$

is a contraction provided $\operatorname{deg}(f)^{k}>|A|$.

Proof. We may assume $|B| \geq 4$. Consider any 4-tuple $B_{0} \subset B$. Since $|P(f)|=3$, we have a point $b \in B_{0}-V\left(f^{k}\right)$. Then $f^{-k}(b)$ is disjoint from $C\left(f^{k}\right)$, and $\left|f^{-k}(b)\right|=\operatorname{deg}(f)^{k}>|A|$, so we cannot have $f^{-k}\left(B_{0}\right) \subset$ $A \cup C\left(f^{k}\right)$.

Factorization. For later use, we record the following fact. Suppose we have a factorization $F=F_{1} \circ F_{2}$. The pullback map can then be factored as

$$
\mathcal{T}_{B} \stackrel{\sigma_{F_{1}}}{\longrightarrow} \mathcal{T}_{C} \stackrel{\sigma_{F_{2}}}{\longrightarrow} \mathcal{T}_{A},
$$

where $C=F_{2}(A) \cup V\left(F_{2}\right)$.

Combinatorial equivalence. Finally we formulate the connection between fixed points on Teichmüller space and rational maps, following Thurston.

Let $F$ and $G$ be a pair of postcritically finite branched coverings of $\mathbb{P}^{1}$. An orientation-preserving homeomorphism of pairs

$$
\phi:\left(\mathbb{P}^{1}, P(F)\right) \rightarrow\left(\mathbb{P}^{1}, P(G)\right)
$$


gives a combinatorial equivalence between $F$ and $G$ if there is a second homeomorphism $\psi$, isotopic to $\phi$ rel $P(F)$, making the diagram

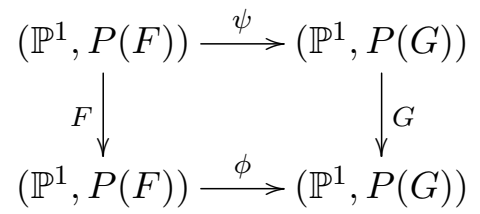

commute. Since $X=P(F)$ is forward invariant, $F$ determines a holomorphic map

$$
\sigma_{F}: \mathcal{T}_{X} \rightarrow \mathcal{T}_{X}
$$

The following result follows readily from the definitions (cf. [DH, Prop. $2.3])$ :

Proposition 3.3 A point $[Y] \in \mathcal{T}_{X}$ is fixed by $\sigma_{F}$ if and only if there exists a rational map $f$ with $P(f)=Y$ such that the marking homeomorphism

$$
\phi:\left(\mathbb{P}^{1}, X\right) \rightarrow\left(\mathbb{P}^{1}, Y\right)
$$

gives a combinatorial equivalence between $F$ and $f$.

\section{Prescribed dynamics on $P(f)$}

In this section we prove Theorem 1.3 . That is, given a finite set $X \subset \mathbb{P}^{1}$ with $|X| \geq 3$, and a map $F: X \rightarrow X$, we will construct a sequence of rigid rational maps $f_{n}$ such that $P\left(f_{n}\right) \rightarrow X$ and

$$
f_{n}\left|P\left(f_{n}\right) \rightarrow F\right| X
$$

This means that for all $n \gg 0$, we can find homeomorphisms $\phi_{n}$ of $\mathbb{P}^{1}$ such that $\phi_{n} \rightarrow \mathrm{id}, \phi_{n}(X)=P\left(f_{n}\right)$, and $\phi_{n}$ conjugates $F \mid X$ to $f_{n} \mid P\left(f_{n}\right)$.

The setup. Let $h$ be a quadratic rational map with $J(h)=\mathbb{P}^{1}$ and

$$
P(h)=\{0,1, \infty\} .
$$

(Explicitly, we can take $\left.h(z)=(2 / z-1)^{2}\right)$. Note that $V\left(h^{n}\right)=P(h)$ for all $n \geq 2$. The map $h$ is expanding in the associated orbifold metric on $\mathbb{P}^{1}$ (see e.g. [Mil1, Thm 19.6], [Mc, App. A]).

It is convenient to normalize so that $X$ contains $P(h)$. Let $g$ be a polynomial fixing 0 and 1 , such that its finite critical values $V_{0}(g)$ coincide with $X-P(h)$. (Such a polynomial exists by Lemma 2.2). Then

$$
V\left(g \circ h^{n}\right)=V(g) \cup g\left(V\left(h^{n}\right)\right)=X
$$


for all $n \geq 2$. For later convenience, we also choose $g$ such that

$$
\operatorname{deg}(g)=3 \text { if }|X|=4
$$

(I.e. if $X=\{0,1, \infty, a\}$, we take $g$ to be a cubic polynomial with $V_{0}(g)=$ $\{a\}$.

Approximation by branched covers. Since $J(h)=\mathbb{P}^{1}$, the set $\bigcup_{n} h^{-n}(x)$ is dense for any $x \in \mathbb{P}^{1}$. Using this fact, we can construct a sequence of homeomorphisms

$$
\phi_{n}:\left(\mathbb{P}^{1}, X\right) \rightarrow\left(\mathbb{P}^{1}, X_{n}\right),
$$

with $\phi_{n} \rightarrow$ id, such that

$$
F\left|X=g \circ h^{n} \circ \phi_{n}\right| X
$$

for all $n$. To do this we first pick, for each $x \in X$, a nearby point $x^{\prime}$ such that $g \circ h^{n}\left(x^{\prime}\right)=F(x)$ and such that the map $x \mapsto x^{\prime}$ is injective; set $X_{n}=\left\{x^{\prime}: x \in X\right\}$. Then, we choose a homeomorphism $\phi_{n}$ close to the identity that moves $x$ to $x^{\prime}$ for all $x \in X$. The larger $n$ is, the closer we can take $x^{\prime}$ to $x$, and hence the closer we can take $\phi_{n}$ to the identity.

Construction of rational maps. Next we observe that

$$
F_{n}=g \circ h^{n} \circ \phi_{n}:\left(\mathbb{P}^{1}, X\right) \rightarrow\left(\mathbb{P}^{1}, X\right)
$$

is a smooth branched covering map with $P\left(F_{n}\right)=X$.

Theorem 1.3 follows from:

Theorem 4.1 For all $n \gg 0, F_{n}$ is combinatorially equivalent to a rational map $f_{n}$; and suitably normalized, we have $f_{n}\left|P\left(f_{n}\right) \rightarrow F\right| X$.

Proof. Using the maps $\phi_{n}$, we can regard $X_{n}$ as points in $\mathcal{T}_{X}$ such that $X_{n} \rightarrow X$. By construction, we have

$$
\sigma_{F_{n}}(X)=X_{n},
$$

and $d\left(X_{n}, X\right) \rightarrow 0$. We wish to control the contraction of $\sigma_{F_{n}}$, and produce a fixed point close to $X$. That is, to complete the proof it suffices to show there are point configurations $P_{n} \rightarrow X$ such that $\sigma_{F_{n}}\left(P_{n}\right)=P_{n}$. For then, by Proposition 3.3 , we have a corresponding sequence of rational maps satisfy $P\left(f_{n}\right)=P_{n}$, and the marking homeomorphisms transport $F \mid X$ to $f_{n} \mid P\left(f_{n}\right)$.

Choose $k$ such that $\operatorname{deg}(h)^{k}=2^{k}>|X|+3$. The crux of the matter is the factorization

$$
F_{n}=\left(g \circ h^{k}\right) \circ\left(h^{n-k} \circ \phi_{n}\right),
$$


valid for all $n \geq k$. From this we obtain a factorization of $\sigma_{F_{n}}$ as

$$
\mathcal{T}_{X} \stackrel{\sigma_{g}}{\longrightarrow} \mathcal{T}_{B} \stackrel{\sigma_{h^{k}}}{\longrightarrow} \mathcal{T}_{A} \stackrel{\sigma_{h^{n-k_{o}} \phi_{n}}}{\longrightarrow} \mathcal{T}_{X}
$$

see equation $(3.2)$. In this factorization, we have

$$
A=h^{n-k}\left(\phi_{n}(X)\right) \cup V\left(h^{n-k}\right) .
$$

Since $\left|V\left(h^{n-k}\right)\right| \leq|P(h)|=3$, we have $\operatorname{deg}(h)^{k}=2^{k}>3+|X| \geq|A|$, and hence $\sigma_{h^{k}}$ is a contraction by Proposition 3.2. Thus $\sigma_{g \circ h^{k}}=\sigma_{h^{k}} \circ \sigma_{g}$ is also a contraction. The amount of contraction at $P \in \mathcal{T}_{X}$ varies continuously with $P$. Since the ball of radius 2 about $X$ in $\mathcal{T}_{X}$ is compact, we can find a constant $\lambda$, independent of $n$, such that

$$
d(P, X) \leq 2 \Longrightarrow\left\|D \sigma_{F_{n}}(P)\right\| \leq\left\|D \sigma_{g \circ h^{k}}(P)\right\| \leq \lambda<1 .
$$

Let $X_{n}^{i}=\sigma_{F_{n}}^{i}(X)$, and let $\epsilon_{n}=d\left(X, X_{n}\right)$. For all $n \gg 0$, we have $\epsilon_{n} \ll(1-\lambda)$. Under this assumption, we can prove by induction that:

(i) $d\left(X_{n}^{i}, X\right) \leq 1$, and hence

(ii) $d\left(X_{n}^{i}, X_{n}^{i-1}\right) \leq \epsilon_{n} \lambda^{i}$.

From this it follows that $X_{n}^{i}$ converges, as $i \rightarrow \infty$, to a fixed point $P_{n}$ of $\sigma_{F_{n}}$ with $d\left(X, P_{n}\right) \leq \epsilon_{n}(1-\lambda)^{-1}$. Since $\epsilon_{n} \rightarrow 0$, this completes the proof.

Proof of Theorem 1.3. We just need to verify that $f_{n}$ is rigid. But if $f_{n}$ is a flexible Lattès example, then $\left|P\left(f_{n}\right)\right|=|X|=4$ and hence $\operatorname{deg}(g)=3$ by condition (4.1). Moreover $\operatorname{deg}\left(f_{n}\right)$ is a square, contradicting the fact that $\operatorname{deg}\left(F_{n}\right)=\operatorname{deg}(g) \operatorname{deg}(h)^{n}=3 \cdot 2^{n}$.

Algorithmic solution. We have used the construction above as the basis for a practical computer program that solves the approximation problem addressed by Theorem 1.3. The iteration does not quite take place on Teichmüller space; rather, we arrange that $P_{n}$ is always close enough to $X$ that there is a unique homeomorphism of $\mathbb{P}^{1}$ close to the identity sending $P_{n}$ to $X$.

\section{Solution to a Hurwitz problem}

In this section we establish the existence of polynomials and rational maps with constrained branch data. This will enable us to strengthen Theorem 1.3 by prescribing local degrees at points of $X$ as discussed in $\$ 6$.

Our main result is: 
Theorem 5.1 Let $\mathcal{P}=\left(P_{1}, \ldots, P_{n}\right)$ be a finite list of partitions. Then $\mathcal{P}$ can be extended to the passport of a polynomial if $n \geq 2$, and to the passport of a rational map if $n \geq 3$.

Partitions. A partition $P$ of $d \geq 0$ is a list of positive integers $\left(p_{1}, \ldots, p_{s}\right)$ such that $\sum p_{i}=d$. The trivial partition has $p_{i}=1$ for $i=1, \ldots, d$.

Given a second partition $P^{\prime}=\left(p_{1}^{\prime}, \ldots, p_{t}^{\prime}\right)$ of $d^{\prime}=\sum p_{i}^{\prime}$, we let

$$
P+P^{\prime}=\left(p_{1}, \ldots, p_{s}, p_{1}^{\prime}, \ldots, p_{t}^{\prime}\right)
$$

denote the combined partition of $d+d^{\prime}$.

Passports. A passport of degree $d$ is a finite list $\mathcal{P}=\left(P_{1}, \ldots, P_{n}\right)$ of nontrivial partitions of $d$. For both partitions and passports, repetitions are allowed and the order in which elements appear is unimportant. We set

$$
c(\mathcal{P})=\sum_{i}\left(d-\left|P_{i}\right|\right) .
$$

Rational maps. For any $y$ in the target of a rational map $f$ we have a partition $P(f, y)$ of $d=\operatorname{deg}(f)$ given by:

$$
\sum_{f(x)=y} \operatorname{mult}(f, x) .
$$

The passport of $f$ is the collection of partitions

$$
\mathcal{P}(f)=\left(P\left(f, v_{1}\right), \ldots, P\left(f, v_{n}\right)\right)
$$

arising from the critical values $\left\{v_{1}, \ldots, v_{n}\right\}$ of $f$. (The other points in the target of $f$ yield trivial partitions.) The number of critical points of $f$ mapping to a given point $y$, counted with multiplicity, is $d-|P(f, y)|$. Hence

$$
c(\mathcal{P}(f))=2 d-2 .
$$

Branched coverings. The passport of a smooth branched covering map $F: S^{2} \rightarrow S^{2}$ is defined similarly. As is well known, any passport that can be realized topologically can be realized geometrically. More precisely, we have:

Proposition 5.2 Let $F: S^{2} \rightarrow S^{2}$ be a branched covering with $V(F)=$ $\left\{v_{1}, \ldots, v_{n}\right\}$, and let $X=\left\{x_{1}, \ldots, x_{n}\right\} \subset \mathbb{P}^{1}$. Then there exists a rational map $f: \mathbb{P}^{1} \rightarrow \mathbb{P}^{1}$ with $\operatorname{deg}(f)=\operatorname{deg}(F)$ and $V(f)=X$ such that

$$
P\left(f, x_{i}\right)=P\left(F, v_{i}\right) \text { for } i=1, \ldots, n .
$$

In particular, $\mathcal{P}(f)=\mathcal{P}(F)$. 
Proof. Choose an orientation-preserving diffeomorphism $\phi: S^{2} \rightarrow \mathbb{P}^{1}$ such that $\phi\left(v_{i}\right)=x_{i}$ for $i=1, \ldots, n$. Pulling the complex structure on $\mathbb{P}^{1}$ back to $S^{2}$ via $\phi \circ F$, and applying the uniformization theorem, we obtain a homeomorphism $\psi: S^{2} \rightarrow \mathbb{P}^{1}$ such that $f=\phi \circ F \circ \psi^{-1}$ is a holomorphic branched covering, and hence a rational map (cf. [Thom]).

Hurwitz problem. The Hurwitz problem is to characterize the passports that arise from branched coverings of $S^{2}$. A complete solution is not known; for background, see e.g. [LZ, Ch. 5]. Theorem 5.1 addresses a variant of this problem where we allow the partitions to be extended.

Extensions. A partition $Q$ extends $P$ if $Q=P+P^{\prime}$ for some partition $P^{\prime}$. (For example, $1+3+5+7=16$ is an extension of $3+7=10$.)

Our main interest is in finite collections of partitions $\mathcal{P}=\left(P_{1}, \ldots, P_{n}\right)$, with repetitions allowed. In this setting we say that $\mathcal{Q}$ extends $\mathcal{P}$ if, when suitably ordered, we have $\mathcal{Q}=\left(Q_{1}, \ldots, Q_{n}\right)$ and $Q_{i}$ extends $P_{i}$ for $i=$ $1, \ldots, n$. In particular, if $\mathcal{Q}$ extends $\mathcal{P}$ then $|\mathcal{Q}|=|\mathcal{P}|$.

Polynomials. When $g: \mathbb{C} \rightarrow \mathbb{C}$ is a polynomial, its passport $\mathcal{P}(g)$ is defined as the list of partitions $P\left(g, v_{i}\right)$ coming from the finite critical values of $g$.

The passports of polynomials are easily described. In fact, by EKS, Prop. 5.2] we have:

Theorem 5.3 A passport $\mathcal{P}=\left(P_{1}, \ldots, P_{n}\right)$ of degree $d$ arises from a polynomial $g$ if and only if

$$
c(\mathcal{P})=\sum_{i}\left(d-\left|P_{i}\right|\right)=d-1 .
$$

The equation above is necessary because $g$ has $d-1$ critical points. Applying Proposition 5.2, we obtain:

Corollary 5.4 Let $X \subset \mathbb{C}$ be a finite set such that $1 \leq|X|<d$. Then there exists a polynomial $g$ of degree $d$ whose critical values coincide with $X$.

Corollary 5.5 Let $\mathcal{P}=\left(P_{1}, \ldots, P_{n}\right)$ be a collection of partitions with $n \geq 2$. Then $\mathcal{P}$ can be extended to the passport of a polynomial of degree $d$ for all $d$ sufficiently large.

Proof. It will be convenient to use exponential notation for repeated integers (so $\left(1^{d}\right)$ is a partition of $d$ ).

First, extend the partitions in $\mathcal{P}$ so they are all nontrivial partitions of the same integer $d$. Then $\mathcal{P}$ is a passport. If we extend $P_{i}$ to $P_{i}+(1)$ for 
all $i$, then $d$ increases by 1 but $c(\mathcal{P})$ remains the same. Thus after a further extension of $\mathcal{P}$, we can assume that $d-1 \geq c(\mathcal{P})$. If equality holds, we are done.

Otherwise, extend $P_{i}$ to $P_{i}+(3)$ for $i=1,2$, and to $P_{i}+(1,1,1)$ for $i \geq 3$. Then $d$ increases by 3 while $c(\mathcal{P})$ increases by 4 . By repeating this type of extension until equality holds in equation (5.2), we obtain an extension of $\mathcal{P}$ that arises from a polynomial of degree $d$.

Finally, suppose $\mathcal{P}$ is the passport of a polynomial of degree $d$. To complete the proof, we will show that $\mathcal{P}$ can be extended to a polynomial passport of degree $d+k$ for any $k \geq 2$. To see this, just extend $P_{1}$ to $P_{1}+(k)$, $P_{2}$ to $P_{2}+\left(2,1^{k-2}\right)$, and $P_{i}$ to $P_{i}+\left(1^{k}\right)$ for $i \geq 3$.

Theorem 5.6 Any collection of partitions $\mathcal{P}=\left(P_{1}, \ldots, P_{n}\right)$ with $n \geq 3$ can be extended to the passport of a rational map.

Proof. We divide the proof into two cases.

Case I. Assume $n \geq 4$. Let $\mathcal{P}_{1}=\left(P_{1}, P_{2}\right)$ and let $\mathcal{P}_{2}=\left(P_{3}, \ldots, P_{n}\right)$. By Corollary 5.5, we may assume that $\mathcal{P}_{1}$ and $\mathcal{P}_{2}$ are passports of polynomials $g_{1}$ and $g_{2}$ of the same degree $d$.

The complex plane can be naturally completed to a closed disk

$$
D \cong \mathbb{C} \cup S^{1}
$$

by adding a circle to represent the rays in $T_{\infty} \widehat{\mathbb{C}}$. Then each polynomial $g_{i}$ extends continuously to a proper map $D_{i}$ of degree $d$ on $D$, satisfying $D_{i}(x)=d x$ for all $x \in S^{1} \cong \mathbb{R} / \mathbb{Z}$.

Now construct a branched covering $F: S^{2} \rightarrow S^{2}$ by gluing together two copies of $D$ to obtain a sphere, and then setting $F=D_{1}$ on the first copy and $F=D_{2}$ on the second. Then $\mathcal{P}(F)=\mathcal{P}$ by construction, and $F$ can be replaced by a rational map by Proposition 5.2, and the proof in this case is complete.

Case II. Now assume that $n=|\mathcal{P}|=3$. We will construct a rational map with $V(f)=\{0,1, \infty\}$ such that $\mathcal{P}(f)$ extends $\mathcal{P}$.

For convenience, let us index the elements $P_{i}$ of $\mathcal{P}$ by $i \in I=\{0,1, \infty\}$. After replacing $P_{i}$ by the extension $P_{i}+(2)$, if necessary, we may assume that every partition in $\mathcal{P}$ is nontrivial.

To construct $f$, it suffices to give the topological data of a dessin d'enfant $D \subset \mathbb{C}[\mathrm{Sn}],[\mathrm{LZ}]$. A dessin is connected graph with vertices of two colors, embedded in the plane, arising as the preimage of the interval $[0,1]$ under 


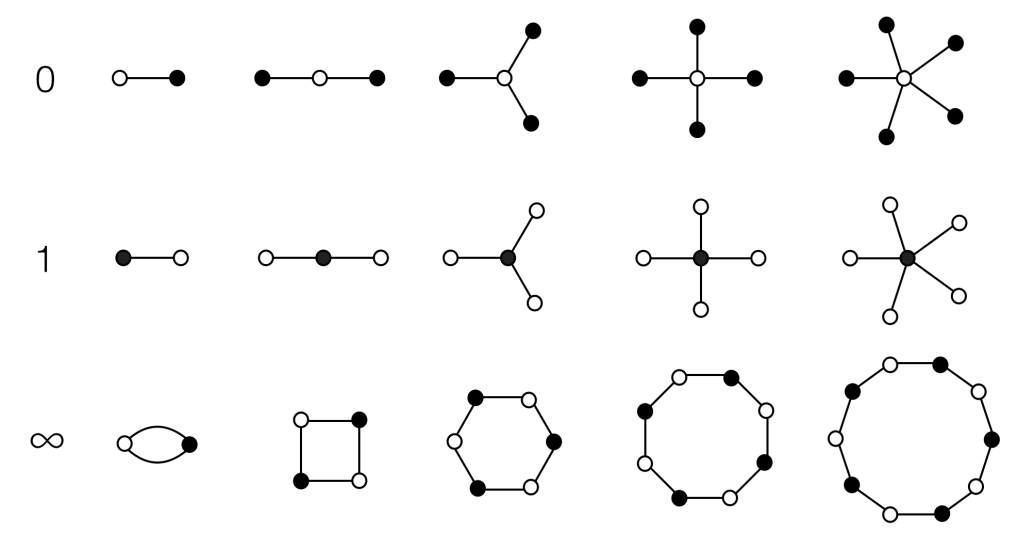

Figure 1: Model dessins $D_{0}(m), D_{1}(m)$, and $D_{\infty}(m)$ are drawn in rows for $1 \leq$ $m \leq 5$.

a branched covering $F: \mathbb{P}^{1} \rightarrow \mathbb{P}^{1}$ with $V(F) \subset\{0,1, \infty\}$. We adopt the convention that the white vertices of $D$ map to 0 and the black vertices map to 1 . The components of $\mathbb{P}^{1}-D$ are called the faces of $D$, and each face contains a unique point $z$ such that $F(z)=\infty$.

Consider, for each $m \geq 1$, the three types of model dessins

$$
D_{0}(m), \quad D_{1}(m), \quad \text { and } \quad D_{\infty}(m)
$$

shown in Figure 1. (These graphs correspond to the rational maps $f_{0}(z)=$ $z^{m}, f_{1}(z)=z^{m}+1$ and $f_{\infty}(z)=\left(z^{m}+z^{-m}+2\right) / 4$. $)$ The barycenter $z$ of $D_{i}(m)$ is a vertex for $i=0$ or $i=1$, and it lies in a face if $i=\infty$. In all three cases, $F(z)=i$ and $\operatorname{mult}(F, z)=m$.

Let $P_{0}=\left(a_{1}, \ldots, a_{n}\right)$, and let $G_{0} \subset \mathbb{C}$ be a planar graph with $n$ components, such that its $j$ th component is isomorphic to $D_{0}\left(a_{j}\right)$. Such a graph is unique up to planar isotopy. Construct $G_{1} \subset \mathbb{C}$ and $G_{\infty} \subset \mathbb{C}$ similarly, using the partitions $P_{1}, P_{\infty}$ and the models $D_{1}(m), D_{\infty}(m)$. When constructing $G_{\infty}$, we take care not to nest two components of type $D_{\infty}(m)$. It is easy to arrange that the graphs $G_{i}$ are contained in disjoint disks in the plane. Let $G=G_{0} \cup G_{1} \cup G_{2}$. Then every vertex of $G$ is incident to the unique unbounded component $U$ of $\mathbb{C}-G$.

To obtain $D$, we add new edges between vertices of opposite colors to make $G$ connected. In the process we take care not to alter the valence at the center of each component of $G$. This can be done in many ways.

For example, begin by introducing a new edge $[b, w] \subset U$, with one white vertex $w$ and one black vertex $b$. Then connect each component of $G_{0}$ to $w$, 


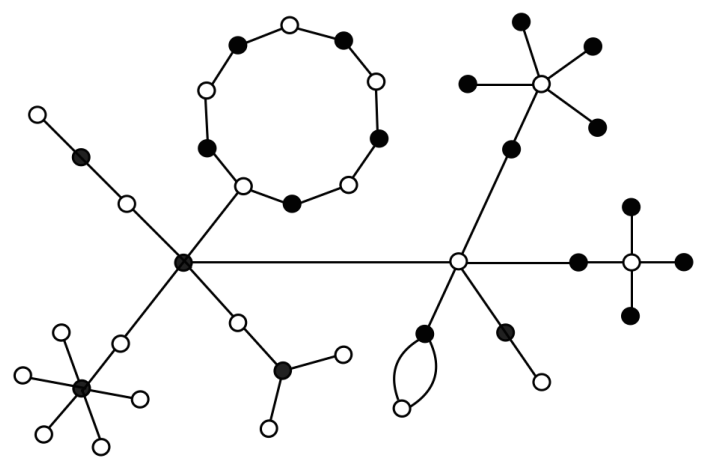

Figure 2: A dessin d'enfant $D$ obtained by connecting the components of $G$ to a single edge $[b, w]$, which is drawn in the center. All four components of $G_{0}$ are connected to $w$; all four components of $G_{1}$ are connected to $b$; and one component of $G_{\infty}$ is connected to $w$ while the other is connected to $b$. The corresponding rational map $f$ has degree 42 .

connect each component of $G_{1}$ to $b$, and connect each component of $G_{\infty}$ to either $w$ or $b$ as in Figure2, making sure that these new edges do not cross.

The resulting connected graph $D \subset \mathbb{C}$ is then a dessin d'enfant for a rational map $f$ with $V(f)=\{0,1, \infty\}$. By the construction of the graph $G_{i}$, the partition $\mathcal{P}(f, i)$ extends $P_{i}$ for each $i \in V(f)$, and hence $\mathcal{P}(f)$ extends $\mathcal{P}$.

Proof of Theorem 5.1. The statements for polynomials and for rational maps are covered by Corollary 5.5 and Theorem 5.6 respectively.

Remarks and references. The proof of Theorem 5.6 for $n \geq 4$ is based on the fact that the passports for a pair of polynomials of degree $d$ can be combined to give the passport of a rational map. This result also appears in [EKS, Remark on p. 785] and [Bar, Prop. 10]. The branched covering built from a pair of polynomials is called their formal mating in [Tan].

\section{Prescribed critical points in $P(f)$}

In this section we strengthen Theorem 1.3 by showing that we can construct a rational map with prescribed critical points in $P(f) \approx X$. We will also give a similar result for polynomials. 
Multiplicities. To make the first statement precise, recall that $f$ has a critical point of order mult $(f, x)-1$ at each point $x \in \mathbb{P}^{1}$. We can regard the multiplicity as a map $\operatorname{mult}(f): \mathbb{P}^{1} \rightarrow \mathbb{Z}_{+}=\{1,2,3, \ldots\}$.

Our aim is to show:

Theorem 6.1 Let $F: X \rightarrow X$ and $M: X \rightarrow \mathbb{Z}_{+}$be arbitrary maps defined on a finite set $X \subset \mathbb{P}^{1}$ with $|X| \geq 3$. Then there exists a sequence of rigid, postcritically finite rational maps $f_{n}$ such that $\left|P\left(f_{n}\right)\right|=|X|$,

$$
P\left(f_{n}\right) \rightarrow X, \quad f_{n}\left|P\left(f_{n}\right) \rightarrow F\right| X, \quad \text { and } \operatorname{mult}\left(f_{n}\right)\left|P\left(f_{n}\right) \rightarrow M\right| X
$$

as $n \rightarrow \infty$.

Proof. The argument is a modification of the proof of Theorem 1.3 given in 84 .

As in that section, the construction is based on a pair of rational maps $g$ and $h$. Let $h$ be a quadratic rational map with $J(h)=\mathbb{P}^{1}$ and $|P(h)|=3$.

To construct $g$, we first associate to each $x \in X$ the partition

$$
P_{x}=\left(M\left(y_{1}\right), \ldots, M\left(y_{n}\right)\right)
$$

where $F^{-1}(x)=\left\{y_{1}, \ldots, y_{n}\right\} \subset X$. Then $\sum_{X}\left|P_{x}\right|=|X|$. (Note that if $x \notin F(X)$, then $P_{x}$ is the empty partition and $\left|P_{x}\right|=0$.)

Let $P_{x}^{\prime}=P_{x}+(1)$ be the partition obtained by padding $P_{x}$ with an extra 1 at the end (notation as in equation (5.1)), so $\left|P_{x}^{\prime}\right|=\left|P_{x}\right|+1$. By Proposition 5.2 and Theorem 5.6, there is a rational map $g$ with critical values $V(g)=X$ such that $P(g, x)$ extends $P_{x}^{\prime}$ for all $x \in X$. Since $|X| \geq 3$, we then have

$$
\left|g^{-1}(X)\right|=\sum_{X}|P(g, x)| \geq \sum_{X}\left|P_{x}^{\prime}\right|=2|X| \geq|X|+3 .
$$

Next, we construct an injective map

$$
\iota: X \rightarrow g^{-1}(X) \subset \mathbb{P}^{1}
$$

such that

$$
F(y)=g(\iota(y)) \text { and } \quad M(y)=\operatorname{mult}(g, \iota(y))
$$

for all $y \in X$. To define $\iota(y)$, let $x=F(y)$ and recall that: $y$ determines a point $M(y) \in \mathcal{P}_{x}$, we have an inclusion $\mathcal{P}_{x} \subset P(g, x)$, and there is a bijection $P(g, x) \cong g^{-1}(x)$ which labels points by their multiplicities. We define $\iota(y)$ to be the image of $M(y)$ under the composition $\mathcal{P}_{x} \subset P(g, x) \cong g^{-1}(x)$. 
Since $|P(h)|=3$ and equation 6.1 holds, we can choose $\alpha \in \operatorname{Aut}\left(\mathbb{P}^{1}\right)$ such that $\alpha(P(h)) \subset g^{-1}(X)-\iota(X)$. Upon replacing $g$ and $\iota$ with $g \circ \alpha$ and $\alpha^{-1} \circ \iota$, equation 6.2 continues to hold, and we then have

$$
P(h) \subset g^{-1}(X)-\iota(X) .
$$

Now recall that $J(h)=\mathbb{P}^{1}$ and hence

$$
X \subset J(h) .
$$

Consequently, for any $x \in X$, the inverse orbit of $\iota(x)$ under $h$ accumulates on every point of $X$. Moreover, if $h^{n}(z)=\iota(x)$, then $\operatorname{mult}\left(h^{n}, z\right)=1$, since $\iota(x) \notin P(h)$. Thus we can find a sequence of injective maps $\phi_{n}: X \rightarrow X_{n}$, converging to the identity, such that

$$
\operatorname{mult}\left(h^{n}\right) \mid X_{n}=1 \text { and } h^{n}\left(\phi_{n}(x)\right)=\iota(x) .
$$

Extend $\left\langle\phi_{n}\right\rangle$ to a sequence of homeomorphisms of $\mathbb{P}^{1}$ converging to the identity as $n \rightarrow \infty$, and let

$$
F_{n}=g \circ h^{n} \circ \phi_{n}:\left(\mathbb{P}^{1}, X\right) \longrightarrow\left(\mathbb{P}^{1}, X\right) .
$$

Then by equation 6.2 , we have

$$
F_{n} \mid X=F \quad \text { and } \operatorname{mult}\left(F_{n}\right)|X=M| X .
$$

Finally, we reiterate the proof of Theorem 4.1 to convert the postcritically finite branched covers $F_{n}$ into rational maps $f_{n}$ with $P\left(f_{n}\right) \rightarrow X$. Since $f_{n}$ and $F_{n}$ are conjugate on their postcritical sets, we then have

$$
f_{n}\left|P\left(f_{n}\right) \rightarrow F\right| X \text { and } \operatorname{mult}\left(f_{n}\right)\left|P\left(f_{n}\right) \rightarrow M\right| X
$$

by equation (6.6). We can also ensure, by our choice of $g$, that $\operatorname{deg}\left(f_{n}\right)$ is not a square, and hence the postcritically finite maps $f_{n}$ are rigid for all $n \gg 0$.

The polynomial case. We conclude by presenting a variation of Theorem 6.1 for polynomials.

Theorem 6.2 Let $F: X \rightarrow X$ and $M: X \rightarrow \mathbb{Z}_{+}$be arbitrary maps defined on a finite set $X \subset \mathbb{C}$ with $|X| \geq 2$. Then there exists a sequence of postcritically finite polynomials $f_{n}$ such that $\left|P_{0}\left(f_{n}\right)\right|=|X|$,

$$
P_{0}\left(f_{n}\right) \rightarrow X, \quad f_{n}\left|P_{0}\left(f_{n}\right) \rightarrow F\right| X, \quad \text { and } \operatorname{mult}\left(f_{n}\right)\left|P_{0}\left(f_{n}\right) \rightarrow M\right| X
$$

as $n \rightarrow \infty$. 
Prescribed Julia sets. For the proof, we will need a polynomial $h$ whose Julia set contains $X$ (to play the role of the rational map $h$ with $J(h)=\mathbb{P}^{1}$ in the proof of Theorem 6.1). It suffices to treat the case where $X \subset \overline{\mathbb{Q}}$, since $\overline{\mathbb{Q}}$ is dense in $\mathbb{C}$.

Lemma 6.3 Given any finite set $X \subset \overline{\mathbb{Q}}$, there is a polynomial $h$ so that

$$
\left|P_{0}(h)\right|=2 \text { and } X \subset J(h) .
$$

Proof. By Theorem 2.3, there is a polynomial $\beta$ with $\beta(X) \cup V_{0}(\beta) \subset\{0,1\}$. We can assume that $\operatorname{deg}(\beta)>1$ and $V_{0}(\beta)=\{0,1\}$ (for example, by taking a Belyi polynomial for a larger set that contains $X)$.

There are two distinct points $a, b \in \beta^{-1}(\{0,1\})$ that are not critical points of $\beta$. Indeed, the set $\beta^{-1}(\{0,1\})$ consists of $2 d$ points, counted with multiplicity, and at most $2\left|C_{0}(f)\right|=2 d-2$ of these are accounted for by critical points.

Let $\alpha \in \operatorname{Aut}(\mathbb{C})$ be an affine transformation sending the ordered pair $(a, b)$ to $(0,1)$, and set $h=\alpha \circ \beta$. We then have:

$$
V_{0}(h)=\{a, b\}, \quad h(\{a, b\}) \subset\{a, b\}, \quad \text { and } \quad h(X) \subset\{a, b\} .
$$

The first two properties imply that $P_{0}(h)=\{a, b\}$, and in particular $P_{0}(h)$ is disjoint from $C_{0}(h)$. It follows that $P_{0}(h)$ is contained in the Julia set of $h$. Since the Julia set is totally invariant and $h(X) \subset\{a, b\}$, we have $X \subset J(h)$ as well.

Proof of Theorem 6.2. We may assume $X \subset \overline{\mathbb{Q}}$. Let $h$ be a polynomial associated to $X$ as in Lemma 6.3. Let $\left(P_{x}^{\prime}: x \in X\right)$ be the family of partitions constructed in the proof of Theorem 6.1, and let $g$ be a polynomial with $V_{0}(g)=X$, provided by Proposition 5.2 and Corollary 5.5, such that $P(g, x)$ extends $P_{x}^{\prime}$ for all $x \in X$. We can now simply repeat the proof of Theorem 6.1, using the mappings $g$ and $h$ to obtain the desired polynomials $f_{n}$.

\section{$7 \quad$ Alternative constructions}

In this section we discuss alternative constructions of rational maps with postcritical sets satisfying $|P(f)| \leq 4$.

The case $|\boldsymbol{P}(\boldsymbol{f})| \leq \mathbf{3}$. The only rational maps with $|P(f)|=2$ are those that are conformally conjugate to $z \mapsto z^{ \pm d}$. Question 1.2 then has a 
negative answer if $X \subset \mathbb{P}^{1}$ has cardinality 2; that is, $F: X \rightarrow X$ is realized by $f: P(f) \rightarrow P(f)$ if and only if $F$ is bijective.

It is easy to see that Question 1.2 has a positive answer whenever $|X|=3$. Indeed, we can first normalize so that $X=\{0,1, \infty\}$. Then, up to reordering the points of $X$, there are only seven possibilities for $F$. A concrete rational map realizing each one is given in Figure 3 .

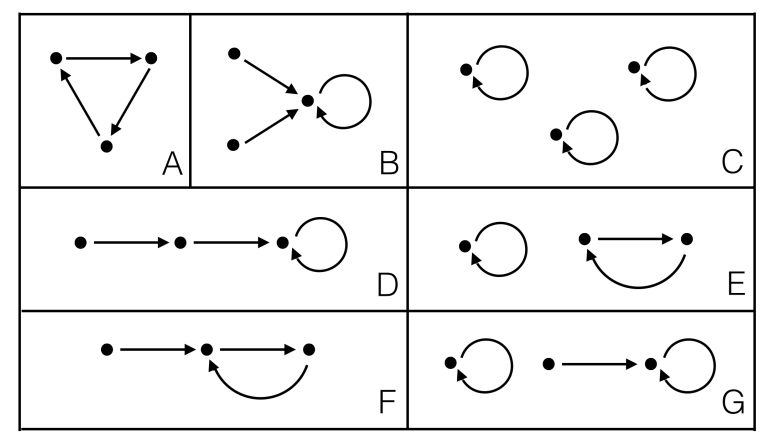

Figure 3: This table contains all seven possible graphs representing maps $X \rightarrow X$ when $|X|=3$. Each graph can be realized by a rational map $f: P(f) \rightarrow P(f)$. Explicit examples are given by $A(z)=1-1 / z^{2} ; B(z)=(z-\alpha)^{3} /(z-1+\alpha)^{3}$ where $\alpha^{2}-\alpha+1=0 ; C(z)=z^{2}(3-2 z) ; D(z)=(1-2 / z)^{2} ; E(z)=z^{2}-1$; and $F(z)=(2 z-1)^{2} /(4 z(z-1))$; and $G(z)=z^{2}-2$. In each case, the degree is minimal among all rational maps realizing the given dynamics on $X$.

The case $|P(f)|=4$ : rigid Lattès maps. Let $Q \subset \mathbb{H}$ denote the set of $\tau$ in the upper halfplane that are quadratic over $\mathbb{Q}$, and let

$$
\lambda: \mathbb{H} \rightarrow \mathbb{H} / \Gamma(2) \cong \mathbb{P}^{1}-\{0,1, \infty\}
$$

be the universal covering map. Rigid Lattès maps are precisely those which are covered by the action of complex multiplication on an elliptic curve $E$ (up to translation by a point of order in $E[2]$ ); see [Mil2, Lemmas 4.3 and 4.4]. Every such elliptic curve has the form $E=\mathbb{C} /(\mathbb{Z} \oplus \mathbb{Z} \tau)$ with $\tau \in Q$. Using these rigid maps, one can explicitly construct $f$ with $P(f)=\{0,1, \infty, z\}$ for all $z$ in the dense set $\lambda(Q) \subset \mathbb{P}^{1}$. On the other hand,

$$
L=\lambda(Q)
$$

is very small subset of $\overline{\mathbb{Q}}$. For example, $L \cap \mathbb{Z}=\{-1,2\}$, since the $j$-invariant

$$
j=\frac{256\left(1-\lambda+\lambda^{2}\right)^{3}}{\lambda^{2}(1-\lambda)^{2}}
$$


is an algebraic integer for all $\lambda \in L$ (see e.g. [Sil, §II.6] for details). So the postcritical sets arising from rigid Lattès examples are insufficient to complete the proof of Theorem 1.1 in the case $|P(f)|=4$.

The case $|P(f)|=4$ : dynamics on moduli space. Our second construction of rational maps with given postcritical sets is based on [Ko. There, the author builds maps on moduli space $g: \mathcal{M}_{0, n} \rightarrow \mathcal{M}_{0, n}$ whose periodic points correspond to postcritically finite rational maps on the Riemann sphere.

For $n=4$, the moduli space $\mathcal{M}_{0,4}$ can be identified with $\mathbb{P}^{1}-\{0,1, \infty\}$, and $g(z)=(1-2 / z)^{2}$ is an example of one such map on moduli space. Each periodic point $x$ of period $m$ gives rise to a postcritically finite polynomial of degree $2^{m}$ with postcritical set $\{0,1, \infty, x\}$. Let $K$ denote the set of all periodic points of $g$ in $\mathbb{P}^{1}-\{0,1, \infty\}$. The set $K$ lies in $\overline{\mathbb{Q}}$, and it is dense in the Julia set $J(g)$. Moreover, we have $J(g)=\mathbb{P}^{1}$ since $g$ is a Lattès map, so $K \subset \mathbb{P}^{1}$ provides a dense set of postcritical sets arising from polynomials.

Interestingly, the intersection $L \cap K$ is finite (where $L$ is defined by equation (7.1)). Indeed, $K$ is a set of bounded Weil height, as a consequence of the existence of a canonical height for $g$ [CS]. But the $j$-invariants of elliptic curves with complex multiplication have no infinite subsets of bounded height [Po, Lemma 3], so neither does $L$.

\section{References}

[Bar] K. Barański. On realizability of branched coverings of the sphere. Topol. Appl. 116(2001), 279-291.

[Bel] G. V. Belyi. On Galois extensions of a maximal cyclotomic field. Math. USSR Izv. 14(1980), 247-256.

[BCT] X. Buff, G. Cui, and Tan L. Teichmüller spaces and holomorphic dynamics. In Handbook of Teichmüller theory. Vol. IV, pages 717756. Eur. Math. Soc., 2014.

[CS] G. S. Call and J. H. Silverman. Canonical heights on varieties with morphisms. Compositio Math. 89(1993), 163-205.

[DH] A. Douady and J. Hubbard. A proof of Thurston's topological characterization of rational maps. Acta Math. 171(1993), 263-297.

[EKS] A. Edmonds, R. Kulkarni, and R. E. Stong. Realizability of branched coverings of surfaces. Trans. Amer. Math. Soc. 282(1984), 773-790. 
[Hub] J. H. Hubbard. Teichmüller Theory, volume II. Matrix Editions, 2016.

[Ko] S. Koch. Teichmüller theory and critically finite endomorphisms. Adv. Math. 248(2013), 573-617.

[LZ] S. K. Lando and A. K. Zvonkin. Graphs on Surfaces and Their Applications. Springer, 2004.

[Mc] C. McMullen. Complex Dynamics and Renormalization, volume 135 of Annals of Math. Studies. Princeton University Press, 1994.

[Mil1] J. Milnor. Dynamics in One Complex Variable, volume 160 of Annals of Math. Studies. Princeton University Press, 2006.

[Mil2] J. Milnor. On Lattès maps. In P. G. Hjorth and C. L. Petersen, editors, Dynamics on the Riemann sphere, pages 9-44. European Math. Soc., 2006.

[Po] B. Poonen. Spans of Hecke points on modular curves. Math. Res. Lett. 8(2001), 767-770.

[Sn] L. Schneps, editor. The Grothendieck theory of dessins d'enfants (Luminy, 1993), volume 200 of London Math. Soc. Lecture Note Ser. Cambridge Univ. Press, 1994.

[Sil] J. Silverman. Advanced topics in the Arithmetic of Elliptic Curves. Springer-Verlag, 1994.

[Tan] Tan L. Matings of quadratic polynomials. Ergod. Th. E Dynam. Sys. 12(1992), 589-620.

[Thom] R. Thom. L'equivalence d'une fonction différentiable et d'un polynôme. Topology 3(1965), 297-307. 